\title{
Antibunching in an optomechanical oscillator
}

\author{
H. Seok ${ }^{1, *}$ and E. M. Wright ${ }^{2}$ \\ ${ }^{1}$ Department of Physics Education, Kongju National University, Gongju 32588, South Korea \\ ${ }^{2}$ College of Optical Sciences and Department of Physics, The University of Arizona, Tucson, Arizona 85721, USA
}

(Received 5 March 2017; published 17 May 2017)

\begin{abstract}
We theoretically analyze antibunching of the phonon field in an optomechanical oscillator employing the membrane-in-the-middle geometry. More specifically, a single-mode mechanical oscillator is quadratically coupled to a single-mode cavity field in the regime in which the cavity dissipation is a dominant source of damping, and adiabatic elimination of the cavity field leads to an effective cubic nonlinearity for the mechanics. We show analytically in the weak-coupling regime that the mechanics displays a chaotic phonon field for small optomechanical cooperativity, whereas an antibunched single-phonon field appears for large optomechanical cooperativity. This opens the door to control of the second-order correlation function of a mechanical oscillator in the weak-coupling regime.
\end{abstract}

DOI: 10.1103/PhysRevA.95.053844

\section{INTRODUCTION}

Cavity optomechanics is a front-line research field in which the motional degrees of freedom of a mechanical oscillator are coupled to optical fields inside an optical cavity, stemming from the interplay of cavity resonance and radiation pressure forces [1-4]. Recent progress in nanoand microfabrication techniques has led to impressive milestones including the cooling of a mechanical oscillator to the motional ground state $[5,6]$, optomechanically induced transparency [7], coherent coupling of optical and mechanical modes [8,9], entanglement between optical and mechanical resonators [10], and optically induced interaction between mechanical oscillators [11]. Cavity optomechanics has numerous applications such as precise measurement of the position of a mirror allowing for gravitational wave detection [12,13], realization of macroscopic quantum objects [14], and as a fundamental platform for exploring coupling to other quantum systems [15-17].

To date almost all experiments on and treatments of cavity optomechanics have been based on linearized optomechanical interactions in the sense that the interaction is linear in both the field and the mechanical variables and is, therefore, based on single photon-phonon interactions [1-4]. The intrinsic optomechanical interaction is, however, nonlinear, which comes to the fore in the single-photon strong-coupling regime. The nonlinear nature of the optomechanical interaction gives rise to a variety of features previously explored in nonlinear quantum optics [18], including photon blockade effects [19], generation of non-Gaussian states [20], and nonclassical antibunched mechanical resonators [21-24].

Currently the single-photon radiation pressure is too low to realize the nonlinear strong-coupling regime in nanofabricated optomechanical systems, making it difficult to observe nonlinear features. To this end, several recent proposals have studied possible nonlinearities in optomechanical setups exploiting, for example, an enhanced optomechanical nonlinearity based on an optomechanical system employing a few optical modes

\footnotetext{
*hseok@kongju.ac.kr
}

[25-28], an intrinsic mechanical nonlinearity [29,30], and the coupling of mechanical systems to a qubit $[31,32]$.

In this paper, we theoretically analyze an approach to producing an antibunched phonon field based on the membrane-in-the-middle geometry and in the weak-coupling regime [33,34]. In particular, a single-mode mechanical oscillator is quadratically coupled to a single-mode cavity field in the regime where the cavity damping is a dominant source of dissipation, resulting in an effective cubic nonlinearity after adiabatic elimination of the cavity field. We show that the mechanical oscillator is coupled to an effective optical reservoir at zero temperature in addition to its own mechanical heat bath at finite temperature. To avoid the difficulties that arise from the multiplicative noise that appears from use of the Heisenberg-Langevin equations, we here employ the Schrödinger picture. Then we demonstrate analytically that the mechanics displays a chaotic phonon field with small multiphoton optomechanical cooperativity, whereas an antibunched single phonon appears for large multiphoton cooperativity.

The remainder of this paper is organized as follows: Sec. II describes the model system, and Sec. III derives the relevant master equation for the mechanical system. In Sec. IV, we employ the complex $P$ representation to investigate the steady-state behaviors of the mechanical oscillator for both the high- and the low-temperature regimes and the appearance of antibunching. Experimental feasibility is discussed in Sec. V, and Sec.VI gives our summary and conclusions.

\section{MODEL SYSTEM}

We consider a membrane-in-the-middle optomechanical system in which the single mode of an optical resonator is quadratically coupled to a single mechanical mode of effective mass $m$ and frequency $\omega_{m}$. The net Hamiltonian governing the optomechanical system is

$$
\hat{H}=\hat{H}_{\mathrm{opt}}+\hat{H}_{\mathrm{mech}}+\hat{H}_{\mathrm{om}}+\hat{H}_{\mathrm{loss}},
$$

where

$$
\hat{H}_{\mathrm{opt}}=\hbar \omega_{c} \hat{a}^{\dagger} \hat{a}+i \hbar\left(\eta e^{-i \omega_{L} t} \hat{a}^{\dagger}-\eta^{*} e^{i \omega_{L} t} \hat{a}\right)
$$


is the Hamiltonian for the single-mode optical field driven by a monochromatic field of frequency $\omega_{L}$ at pumping rate $\eta$, and

$$
\hat{H}_{\text {mech }}=\hbar \omega_{m} \hat{b}^{\dagger} \hat{b}
$$

is the Hamiltonian for the free mechanical mode. The optomechanical interaction is given by

$$
\hat{H}_{\mathrm{om}}=\hbar g_{0} \hat{a}^{\dagger} \hat{a}\left(\hat{b}+\hat{b}^{\dagger}\right)^{2},
$$

where $g_{0}>0$ is the quadratic single-photon optomechanical coupling coefficient, which we choose as positive to avoid any issues of mechanical instability [35]. Finally, $\hat{H}_{\text {loss }}$ describes the interaction of the cavity-field and mechanical modes with their associated reservoirs and accounts for dissipation.

\section{DENSITY OPERATOR FORMALISM}

The Heisenberg-Langevin equations of motion for our problem can involve multiplicative quantum noise in the presence of nonlinear interactions [35]. To circumvent these problems we here explore the dynamics of the optomechanical system in the Schrödinger picture since the equation of motion describing the optomechanical system is then strictly linear in the density operator. The dynamics of the optomechanical system under the influence of thermal fluctuations in the quantum regime can then be described by the master equation [36]

$$
\begin{aligned}
\dot{\tilde{\rho}}= & -\frac{i}{\hbar}\left[\hat{H}_{\mathrm{opt}}+\hat{H}_{\mathrm{mech}}+\hat{H}_{\mathrm{om}}, \tilde{\rho}\right]+\frac{\kappa}{2} \mathcal{D}[\hat{a}] \tilde{\rho} \\
& +\frac{\gamma}{2} \bar{n}_{\mathrm{th}} \mathcal{D}\left[\hat{b}^{\dagger}\right] \tilde{\rho}+\frac{\gamma}{2}\left(\bar{n}_{\mathrm{th}}+1\right) \mathcal{D}[\hat{b}] \tilde{\rho},
\end{aligned}
$$

where $\tilde{\rho}$ is the density operator for the combined optomechanical system, and the dissipation terms $\mathcal{D}[\hat{o}] \tilde{\rho}$ are of the standard Lindblad form

$$
\mathcal{D}[\hat{o}] \tilde{\rho}=\left(2 \hat{o} \tilde{\rho} \hat{o}^{\dagger}-\hat{o}^{\dagger} \hat{o} \tilde{\rho}-\tilde{\rho} \hat{o}^{\dagger} \hat{o}\right) .
$$

These account for damping of the cavity field at decay rate $\kappa$ due to the coupling to a zero-temperature optical reservoir and damping of the mechanical oscillator at decay rate $\gamma$ due to interaction with a mechanical reservoir at temperature $T$. The thermal occupation number of the mechanical bath is denoted $\bar{n}_{\mathrm{th}}=\left[\exp \left(\hbar \omega_{m} / k_{B} T\right)-1\right]^{-1}$.

\section{A. Master equation in the interaction picture}

To proceed it is convenient to introduce the unitary operator $\hat{U}_{1}$, which transforms to a frame rotating at driving frequency $\omega_{L}$ for the cavity field

$$
\hat{U}_{1}=e^{-i \omega_{L} \hat{a}^{\dagger} \hat{a} t},
$$

and the unitary displacement operator $\hat{U}_{2}$, capturing the steadystate mean amplitude of the cavity field resulting from the external pump

$$
\hat{U}_{2}=e^{\left(\alpha \hat{a}^{\dagger}-\alpha^{*} \hat{a}\right)},
$$

with the steady-state intracavity amplitude $\alpha$ given by

$$
\alpha=\frac{\eta}{-i \Delta_{c}+\kappa / 2} \equiv \sqrt{n_{c}} .
$$

Without loss of generality $\alpha$ is here chosen as real by judicious choice of the phase of the pumping rate $\eta$, and
$\Delta_{c}=\omega_{L}-\omega_{c}$ is the detuning of the pump laser from the resonance. The master equation for the transformed density operator $\bar{\rho}=\hat{U}_{2}^{\dagger} \hat{U}_{1}^{\dagger} \tilde{\rho} \hat{U}_{1} \hat{U}_{2}$ then becomes

$$
\begin{aligned}
\dot{\bar{\rho}}= & i \Delta_{c}\left[\hat{a}^{\dagger} \hat{a}, \bar{\rho}\right]-i \omega_{m}^{\prime}\left[\hat{b}^{\dagger} \hat{b}, \bar{\rho}\right]-i g_{0} n_{c}\left[\hat{b}^{\dagger 2}+\hat{b}^{2}, \bar{\rho}\right] \\
& -i g\left[\left(\hat{a}+\hat{a}^{\dagger}\right)\left(\hat{b}^{\dagger}+\hat{b}\right)^{2}, \bar{\rho}\right]-i g_{0}\left[\hat{a}^{\dagger} \hat{a}\left(\hat{b}^{\dagger}+\hat{b}\right)^{2}, \bar{\rho}\right] \\
& +\frac{\kappa}{2} \mathcal{D}[\hat{a}] \bar{\rho}+\frac{\gamma}{2} \bar{n}_{\mathrm{th}} \mathcal{D}\left[\hat{b}^{\dagger}\right] \bar{\rho}+\frac{\gamma}{2}\left(\bar{n}_{\mathrm{th}}+1\right) \mathcal{D}[\hat{b}] \bar{\rho}
\end{aligned}
$$

where $\omega_{m}^{\prime}=\omega_{m}+2 g_{0} n_{c}$ is the shifted frequency of the mechanical oscillator, and $g=g_{0} \sqrt{n_{c}}$. This frequency shift proportional to the intracavity photon number comes from the quadratic optomechanical interaction, as opposed to the displacement of the mechanical equilibrium position, which rises in the case of linear optomechanical coupling.

In the regime in which the mean cavity photon number $n_{c}$ is much larger than the photon fluctuations, the fifth term on the right-hand-side of Eq. (10) may be neglected: This term is a factor $1 / n_{c}$ smaller than the third term and a factor $1 / \sqrt{n_{c}}$ smaller than the fourth term, these also arising from the quadratic interaction. Following this approximation leads to an optomechanical interaction that is linear in the cavity-field operators.

In order to investigate the mechanics in the deep quantum regime, we proceed by assuming that the optomechanical system operates in the resolved-sideband regime, which requires $\omega_{m}^{\prime} \gg \kappa$, and that the external pump is red-detuned by twice the effective mechanical frequency, $\Delta_{c}=-2 \omega_{m}^{\prime}$. Then a further simplification follows by invoking the rotating-wave approximation in the interaction picture implemented by the unitary transformation $\hat{U}_{3}=e^{i\left(\Delta_{c} \hat{a}^{\dagger} \hat{a}-\omega_{m}^{\prime} \hat{b}^{\dagger} \hat{b}\right) t}$, and the resulting master equation becomes

$$
\begin{aligned}
\dot{\rho}= & -i g\left[\hat{a}^{\dagger} \hat{b}^{2}+\hat{b}^{\dagger 2} \hat{a}, \rho\right]+\frac{\kappa}{2} \mathcal{D}[\hat{a}] \rho+\frac{\gamma}{2} \bar{n}_{\mathrm{th}} \mathcal{D}\left[\hat{b}^{\dagger}\right] \rho \\
& +\frac{\gamma}{2}\left(\bar{n}_{\mathrm{th}}+1\right) \mathcal{D}[\hat{b}] \rho,
\end{aligned}
$$

where $\rho=\hat{U}_{3}^{\dagger} \bar{\rho} \hat{U}_{3}$. We note that the third term on the righthand side of Eq. (10) has been neglected on the basis that it is off-resonant and counter-rotating if $g_{0} n_{c} \ll \omega_{m}^{\prime}$, and we have checked numerically that this term is indeed negligible in the weak-coupling regime. Physically the Hamiltonian representing the Schrödinger evolution in Eq. (11) reads

$$
\hat{H}=\hbar g\left(\hat{a}^{\dagger} \hat{b}^{2}+\hat{b}^{\dagger 2} \hat{a}\right)
$$

and is identical to the interaction picture Hamiltonian describing a parametric amplifier in quantum optics and is well known to generate two photons in the subharmonic mode $(\hat{b})$ and destroy a photon in the pump mode ( $\hat{a})$ [37]. It is thus expected that two phonons of the mechanics can be destroyed by creating a single photon which is eventually leaked out the optical resonator by the cavity-field dissipation at rate $\kappa$.

\section{B. Reduced density operator for the mechanics}

In the regime where cavity dissipation is the dominant source of damping, the state of the cavity field tends to approach a coherent state on a time scale of $1 / \kappa$ and thus the density operator describing the optomechanical system can be 
approximated as a product state,

$$
\rho(t) \approx \rho_{o}(t) \otimes \rho_{m}(t)
$$

where $\hat{\rho}_{o}$ is the reduced density operator for the cavity field and $\hat{\rho}_{m}$ is the reduced density operator for the mechanics. One should keep in mind that on a time scale slower than $1 / \kappa$, the dynamics of the optomechanical system is dependent on that of the mechanical oscillator, whereas the dynamics of the cavity field is instantaneously followed by that of the mechanics due to the fast dissipation of the cavity field. Specifically, the reduced density operator for the cavity field describes the vacuum state $\hat{\rho}_{o}=(|0\rangle\langle 0|)_{o}$ in that we are already in the displaced-field picture.

In order to properly eliminate the reduced density operator for the cavity field and to derive the effective master equation for the mechanical oscillator, we follow the approach used for eliminating the density operator for the pump mode of a parametric amplifier in quantum optics or for the cavity field in cavity QED (see, e.g., [38]). The dynamics of the reduced density operator for the mechanics is then described by the effective master equation

$$
\begin{aligned}
\frac{d \rho_{m}}{d t}= & \frac{\Gamma_{\mathrm{opt}}}{2} \mathcal{D}\left[\hat{b}^{2}\right] \rho_{m}+\frac{\gamma}{2} \bar{n}_{\mathrm{th}} \mathcal{D}\left[\hat{b}^{\dagger}\right] \rho_{m} \\
& +\frac{\gamma}{2}\left(\bar{n}_{\mathrm{th}}+1\right) \mathcal{D}[\hat{b}] \rho_{m},
\end{aligned}
$$

where $\Gamma_{\text {opt }}$ is the nonlinear optomechanical damping rate given by

$$
\Gamma_{\mathrm{opt}}=\frac{8 g^{2}}{\kappa} .
$$

Note that this rate is identical to the maximum value of the optomechanical damping rate for the resolved-sideband regime [1]. The first term on the right-hand side of the effective master equation accounts for two-phonon damping of the mechanical oscillator and the damping rate is proportional to the cavity photon number, indicating that the mechanical oscillator experiences the optical reservoir at zero temperature through the cavity field. In other words, the intracavity photon number can be used as a control parameter for the nonlinear optomechanical coupling strength of the mechanics to the optical reservoir. That is, the dynamics and steady-state properties of the mechanical oscillator are affected by two independent heat baths: the optical bath at zero temperature via two-phonon processes and the mechanical bath at finite temperature via one-phonon processes. We remark that Eq. (14) was previously solved in the context of a laser with intracavity two-photon absorption using a generating function from which the photon populations and relevant moments can be obtained $[39,40]$. The solutions given here are in agreement with this analytic solution.

It is convenient to scale time to the inverse of the mechanical decay rate, $\tau=\gamma t$, in terms of which the effective master equation for the mechanics then becomes

$$
\begin{aligned}
\frac{d \rho_{m}}{d \tau}= & \frac{C}{2} \mathcal{D}\left[\hat{b}^{2}\right] \rho_{m}+\frac{1}{2} \bar{n}_{\mathrm{th}} \mathcal{D}\left[\hat{b}^{\dagger}\right] \rho_{m} \\
& +\frac{1}{2}\left(\bar{n}_{\mathrm{th}}+1\right) \mathcal{D}[\hat{b}] \rho_{m},
\end{aligned}
$$

where the multiphoton cooperativity $C$ is given by

$$
C=\frac{\Gamma_{\mathrm{opt}}}{\gamma}=\frac{8 g^{2}}{\gamma \kappa} .
$$

The multiphoton cooperativity is dimensionless and is a measure of the relative coupling strengths of the mechanical oscillator to the cavity-filtered optical bath and mechanical heat bath. A large cooperativity compared to the thermal occupation number $\bar{n}_{\text {th }}$ indicates that mechanical oscillator is more influenced by the optical bath than the mechanical bath and the dynamics of the mechanics is highly nonlinear.

\section{RESULTS}

We now turn to the analytic solution of the master equation for the mechanics in the high- and low-temperature regimes and explore the second-order correlation function for the mechanics, defined as

$$
g^{(2)}(0) \equiv \frac{\left\langle\hat{b}^{\dagger 2} \hat{b}^{2}\right\rangle_{\mathrm{ss}}}{\left\langle\hat{b}^{\dagger} \hat{b}\right\rangle_{\mathrm{ss}}^{2}},
$$

where the subscript "ss" denotes the expectation values taken with respect to the steady-state solution. For this purpose we employ well-known phase-space methods that we now discuss briefly as applied to our case.

\section{A. Phase-space methods}

As is well known, a nonlinear quantum mechanical problem can be mapped into a classical stochastic process with an appropriate phase-space representation. We proceed to derive the equation of motion for the mechanical system in the complex $P$ representation. Expanding the density operator for the mechanics as

$$
\rho_{m}=\int \frac{|\mu\rangle\left\langle v^{*}\right|}{\left\langle\nu^{*} \mid \mu\right\rangle} P(\mu, v) d \mu d \nu
$$

and making use of the quantum correspondence appropriate for the complex $P$ representation [41]

$$
\begin{aligned}
\hat{b} \rho_{m} & \leftrightarrow \mu P(\mu, v), \\
\hat{b}^{\dagger} \rho_{m} & \leftrightarrow\left(v-\frac{\partial}{\partial \mu}\right) P(\mu, v), \\
\rho_{m} \hat{b}^{\dagger} & \leftrightarrow \nu P(\mu, v), \\
\rho_{m} \hat{b} & \leftrightarrow\left(\mu-\frac{\partial}{\partial v}\right) P(\mu, v),
\end{aligned}
$$

the master equation for the mechanics takes the form of the Fokker-Planck equation

$$
\begin{aligned}
\frac{d P(\chi)}{d \tau}= & -\sum_{i} \frac{\partial}{\partial \chi_{i}}[A(\chi)]_{i} P(\chi) \\
& +\frac{1}{2} \sum_{i, j} \frac{\partial}{\partial \chi_{i}} \frac{\partial}{\partial \chi_{j}}[D(\chi)]_{i, j} P(\chi),
\end{aligned}
$$

where $\chi=(\mu, v)^{T}$, the drift vector $A(\chi)$ is given by

$$
A(\chi)=\left(\begin{array}{l}
-\frac{1}{2} \mu-C v \mu^{2} \\
-\frac{1}{2} \nu-C \mu \nu^{2}
\end{array}\right),
$$


and the diffusion matrix $D(\chi)$ is

$$
D(\chi)=\left(\begin{array}{cc}
-C \mu^{2} & \bar{n}_{\mathrm{th}} \\
\bar{n}_{\mathrm{th}} & -C v^{2}
\end{array}\right) .
$$

We remark that Eq. (24) is identical to that for the complex $P$ distribution for a single-mode optical field in a cavity that involves a cubic-nonlinear dispersive medium [42]. We further note that there are two diffusion sources for the complex $P$ distribution function, thermal fluctuations due to the mechanical heat bath represented by the off-diagonal elements of the diffusion matrix, and additional quantum fluctuations due to the optomechanical interaction represented by the diagonal elements. Given the steady-state complex distribution function $P_{s}$ all normally ordered steady-state moments can be calculated as

$$
\left\langle\left(\hat{b}^{\dagger}\right)^{n}(\hat{b})^{n^{\prime}}\right\rangle_{\mathrm{ss}}=\int d \mu\left(\mu^{*}\right)^{n}(\mu)^{n^{\prime}} P_{s}\left(\mu, \mu^{*}\right)
$$

\section{B. High-temperature regime}

In the regime where the thermal fluctuations are the dominant source of diffusion, $\bar{n}_{\text {th }} \gg C$, we are able to neglect quantum fluctuations resulting from the optomechanical interaction so that the diffusion matrix can be approximated as

$$
D(\chi) \approx\left(\begin{array}{cc}
0 & \bar{n}_{\mathrm{th}} \\
\bar{n}_{\mathrm{th}} & 0
\end{array}\right) .
$$

Then setting the left-hand side of Eq. (24) to 0 at steady state and employing the usual potential condition [37], the distribution function is readily found as

$$
P_{s}(\mu, \nu)=\mathcal{N} \exp \left(-\frac{1}{\bar{n}_{\mathrm{th}}} \mu \nu\right) \exp \left(-\frac{C}{\bar{n}_{\mathrm{th}}} \mu^{2} v^{2}\right),
$$

where $\mathcal{N}$ is a normalization constant. Note that this complex $P$ distribution is bounded and well behaved in the domain in which $v=\mu^{*}$, namely, the Glauber-Sudarshan $P$ representation can be used [43]. The corresponding Glauber-Sudarshan $P$ distribution becomes

$$
P_{s}\left(\mu, \mu^{*}\right)=\mathcal{N} \exp \left(-\frac{1}{\bar{n}_{\mathrm{th}}}|\mu|^{2}\right) \exp \left(-\frac{C}{\bar{n}_{\mathrm{th}}}|\mu|^{4}\right) .
$$

From this result we see that for $C \ll 1$ the Glauber-Sudarshan $P$ distribution approaches that for a thermal mixture with occupation number $\bar{n}_{\text {th }}$,

$$
P_{s}\left(\mu, \mu^{*}\right) \approx \frac{1}{\bar{n}_{\mathrm{th}} \pi} \exp \left(-\frac{1}{\bar{n}_{\mathrm{th}}}|\mu|^{2}\right),
$$

as expected in the limit of small multiphoton cooperativity [44]. On the other hand, the Glauber-Sudarshan $P$ distribution can be approximated as

$$
P_{s}\left(\mu, \mu^{*}\right) \approx \frac{2}{\pi^{3 / 2}} \sqrt{\frac{C}{\bar{n}_{\mathrm{th}}}} \exp \left(-\frac{C}{\bar{n}_{\mathrm{th}}}|\mu|^{4}\right)
$$

in the limit of large multiphoton cooperativity, $C \gg 1$.

In Fig. 1 we plot the steady-state mean phonon number obtained from Eq. (27),

$$
\left\langle\hat{b}^{\dagger} \hat{b}\right\rangle_{\mathrm{ss}} \equiv n_{\mathrm{ss}}=-\frac{1}{2 C}+\sqrt{\frac{\bar{n}_{\mathrm{th}}}{\pi C}} \frac{\exp \left(-\frac{1}{4 C \bar{n}_{\mathrm{th}}}\right)}{\operatorname{erfc}\left(\sqrt{\frac{1}{4 C \bar{n}_{\mathrm{th}}}}\right)},
$$

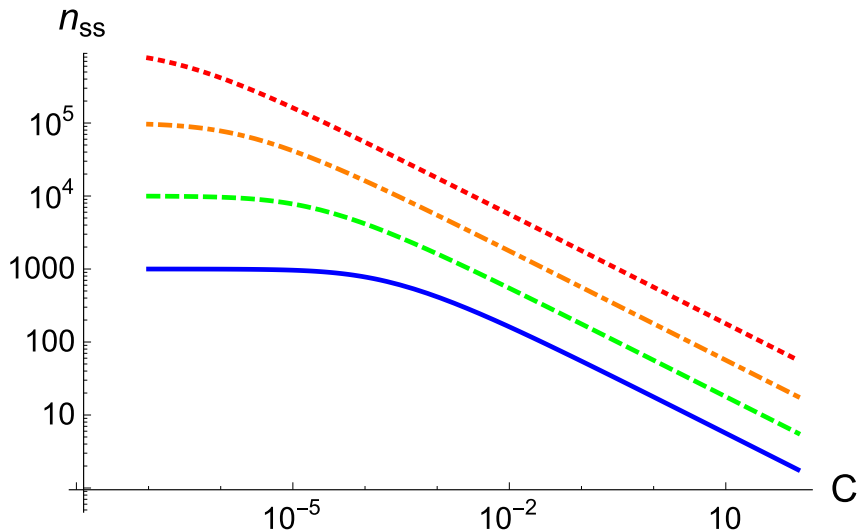

FIG. 1. Steady-state mean phonon number of the mechanics as a function of the multiphoton cooperativity $C$ for different thermal occupation numbers $\bar{n}_{\mathrm{th}} ; \bar{n}_{\mathrm{th}}=10^{6}$ (dotted red line), $\bar{n}_{\mathrm{th}}=10^{5}$ (dotdashed orange line), $\bar{n}_{\mathrm{th}}=10^{4}$ (dashed green line), and $\bar{n}_{\mathrm{th}}=10^{3}$ (solid blue line).

versus the multiphoton cooperativity $C$ for different thermal phonon numbers $\bar{n}_{\text {th }}$. Here $\operatorname{erfc}(x)=1-\operatorname{erf}(x)$ is the complementary error function. The results show that the mechanics, in a thermal state of mean occupation number $\bar{n}_{\text {th }}$ at low multiphoton cooperativity, is cooled down as the multiphoton cooperativity $C$ is increased. Indeed the steady-state mean phonon number approaches

$$
n_{\mathrm{ss}} \approx \sqrt{\frac{\bar{n}_{\mathrm{th}}}{\pi C}}
$$

in the limit of large multiphoton cooperativity, $C \gg 1$.

To investigate further we calculate the second-order correlation function $g^{(2)}(0)$ for the mechanics and plot it in Fig. 2 as a function of the multiphoton cooperativity for different thermal occupation numbers. This figure makes clear that in the regime where $C \ll 1$ the second-order correlation

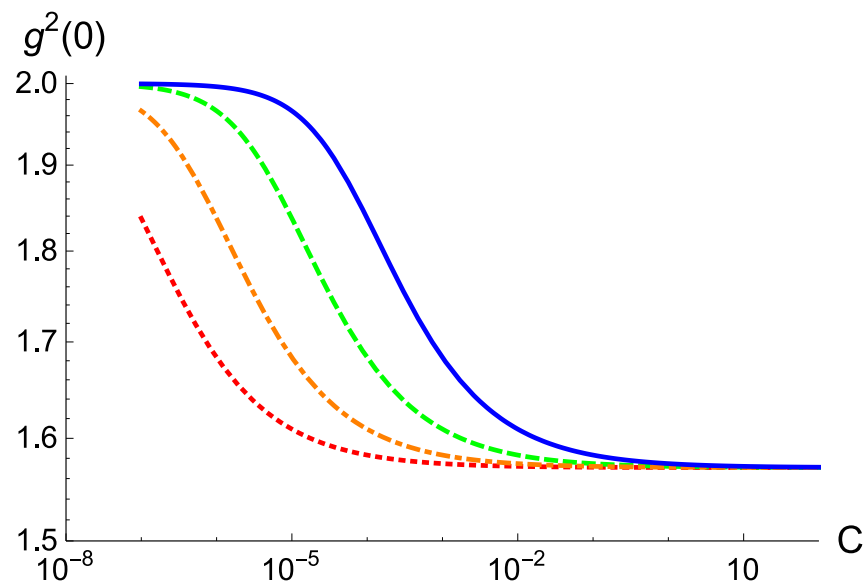

FIG. 2. Steady-state second-order correlation function $g^{(2)}(0)$ in the high-temperature regime as a function of the multiphoton cooperativity $C$ for different thermal occupation numbers $\bar{n}_{\mathrm{th}} ; \bar{n}_{\mathrm{th}}=$ $10^{6}$ (dotted red line), $\bar{n}_{\text {th }}=10^{5}$ (dot-dashed orange line), $\bar{n}_{\text {th }}=10^{4}$ (dashed green line), and $\bar{n}_{\text {th }}=10^{3}$ (solid blue line). One-phonon absorption and emission processes are dominant so that the secondorder correlation function is larger than unity. 


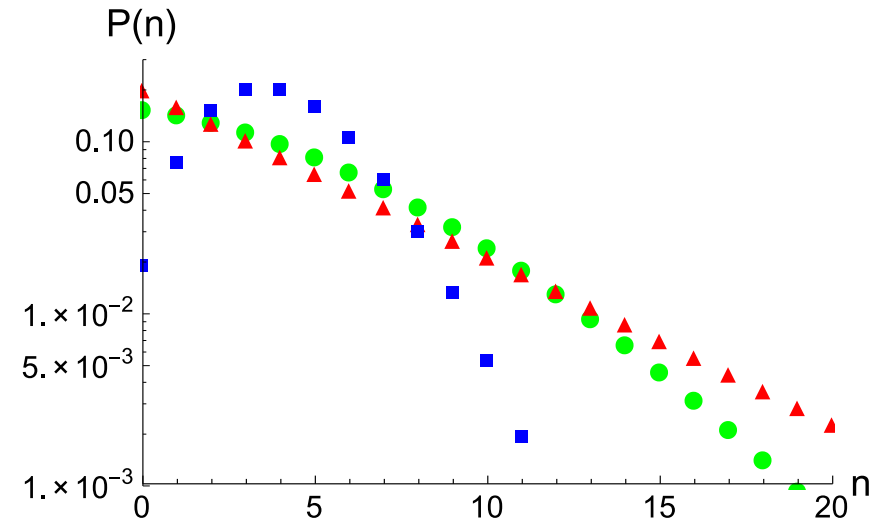

FIG. 3. Steady-state phonon number distribution $P(n)$ of the mechanical oscillator (green circles) for $\bar{n}_{\mathrm{th}}=10^{4}$ and $C=10^{2}$. For comparison, the phonon number distributions of the mechanics in a thermal state (red triangles) and a coherent state (blue squares) with the same mean phonon number are shown.

function $g^{(2)}(0)$ becomes 2, a feature of a thermal state. On the other hand, $g^{(2)}(0)$ approaches $\pi / 2$ for large multiphoton cooperativity, indicating that the steady state of the mechanics is chaotic. This tendency stems from the fact that the linear thermal fluctuations overwhelm the nonlinear two-phonon optomechanical cooling. As a result, the phonon distribution is always bunched in the high-temperature regime, and the variance of the phonon number distribution for the mechanical oscillator is in between those of the mechanics at thermal equilibrium and a coherent state with the same mean phonon number. This is illustrated in Fig. 3, which shows the steadystate phonon number distribution $P(n)$ of the mechanical oscillator (green circles) for $\bar{n}_{\text {th }}=10^{4}$ and $C=10^{2}$, along with the cases of a thermal state (red triangles) and a coherent state (blue squares) for comparison.

\section{Low-temperature regime}

In order to explore the possibility of an antibunched phonon field, a key signature that the mechanical system is in a truly quantum state, we proceed to examine the low-temperature regime. We have obtained the steady-state complex $P$ distribution following the procedures outlined in Ref. [45], but for the sake of clarity of presentation we relegate the details to the Appendix and concentrate on the results here. Specifically, we find that the complex $P$ distribution is given by

$$
\begin{aligned}
P_{s}(\mu, \nu)= & \frac{2 A e^{2 \mu \nu}}{\left(1+2 \bar{n}_{\mathrm{th}}-C\right) \mu \nu}{ }_{2} F_{1}\left(1,1 ; \frac{1+2 \bar{n}_{\mathrm{th}}}{C} ; \frac{\bar{n}_{\mathrm{th}}}{C \mu \nu}\right) \\
& +\frac{2 A e^{2 \mu \nu}}{\bar{n}_{\mathrm{th}}} \sum_{r=1}^{\infty} \frac{(-2 \mu \nu)^{r}}{r r !} \\
& \times{ }_{2} F_{1}\left(1,2+r-\frac{1+2 \bar{n}_{\mathrm{th}}}{C} ; 1+r ; \frac{C \mu \nu}{\bar{n}_{\mathrm{th}}}\right),
\end{aligned}
$$

where ${ }_{2} F_{1}(a, b ; c ; z)$ is the hypergeometric function. The corresponding expression for the steady-state mean phonon number of the mechanics is given by Eq. (A23) and is plotted in Fig. 4 as a function of the multiphoton cooperativity $C$ and for a variety of thermal occupation numbers. These results

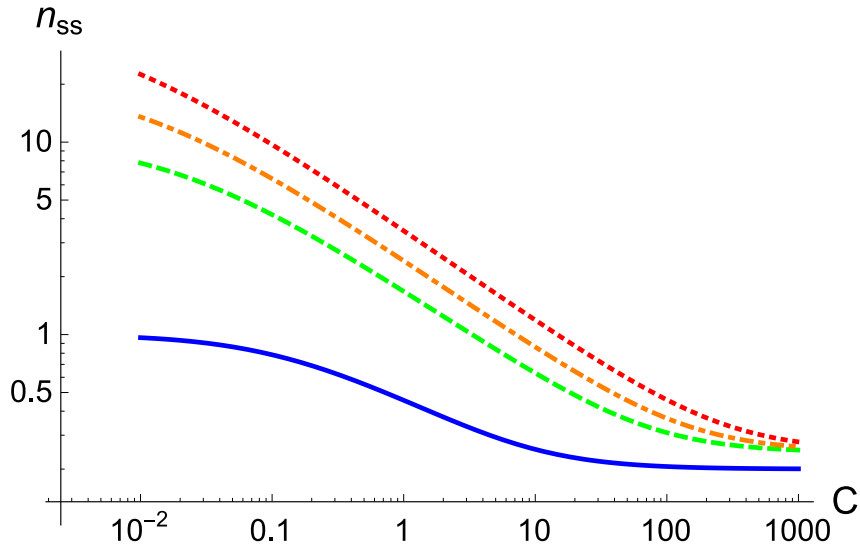

FIG. 4. Steady-state mean phonon number $n_{\mathrm{ss}}$ of the mechanical oscillator as a function of the multiphoton cooperativity $C$ in the low-temperature regime: $\bar{n}_{\mathrm{th}}=1$ (solid blue line), $\bar{n}_{\mathrm{th}}=10$ (dashed green line), $\bar{n}_{\mathrm{th}}=20$ (dot-dashed orange line), and $\bar{n}_{\mathrm{th}}=40$ (dotted red line).

show that the mechanics is cooled down near the motional ground state in the regime where $C \gg 1$. In this regime the optomechanical two-phonon damping is dominant so that only the ground and first excited states are significantly populated (see the steady-state phonon distribution, indicated by blue rhombi, in Fig. 6) and the ground-state population and the population of the first excited state are approximately given by

$$
\begin{aligned}
& P(0) \approx \frac{3 \bar{n}_{\mathrm{th}}+1}{4 \bar{n}_{\mathrm{th}}+1}, \\
& P(1) \approx \frac{\bar{n}_{\mathrm{th}}}{4 \bar{n}_{\mathrm{th}}+1},
\end{aligned}
$$

respectively. These results are in accordance with the numerical calculations based on the Fock-state representation [46].

The expression for the second-order correlation function $g^{(2)}(0)$ of the mechanics is given by Eq. (A24). Figure 5 shows a color-coded plot of the second-order correlation function of the mechanical oscillator as a function of both the mutiphoton cooperativity $(C)$ and the thermal occupation number $\left(\bar{n}_{\mathrm{th}}\right)$. The plot reveals that the phonon distribution of the mechanics is antibunched $\left[g^{(2)}(0)<1\right]$ when $C>2 \bar{n}_{\text {th }}+1$, whereas it is bunched $\left[g^{(2)}(0)>1\right]$ when $C<2 \bar{n}_{\text {th }}+1$. Physically, the mechanics tends to experience one-phonon absorption and emission processes, and its phonon distribution is super-Poissonian, if the mechanical thermal and quantum noise sources are dominant, $C<2 \bar{n}_{\text {th }}+1$. However, in the regime where the optomechanical coupling is stronger than the thermal decoherence, $C>2 \bar{n}_{\text {th }}+1$, the mechanics has a tendency to experience two-phonon absorption and emission processes and its phonon distribution becomes antibunched. When $C \gg 2 \bar{n}_{\text {th }}+1$, the second-order correlation function can be simplified as

$$
g^{(2)}(0)=\frac{2\left(4 \bar{n}_{\mathrm{th}}+1\right)}{C}+O\left(\frac{1}{C^{2}}\right) .
$$

As expected, when $C=2 \bar{n}_{\text {th }}+1$ the steady state of the mechanical oscillator becomes a coherent state with mean 


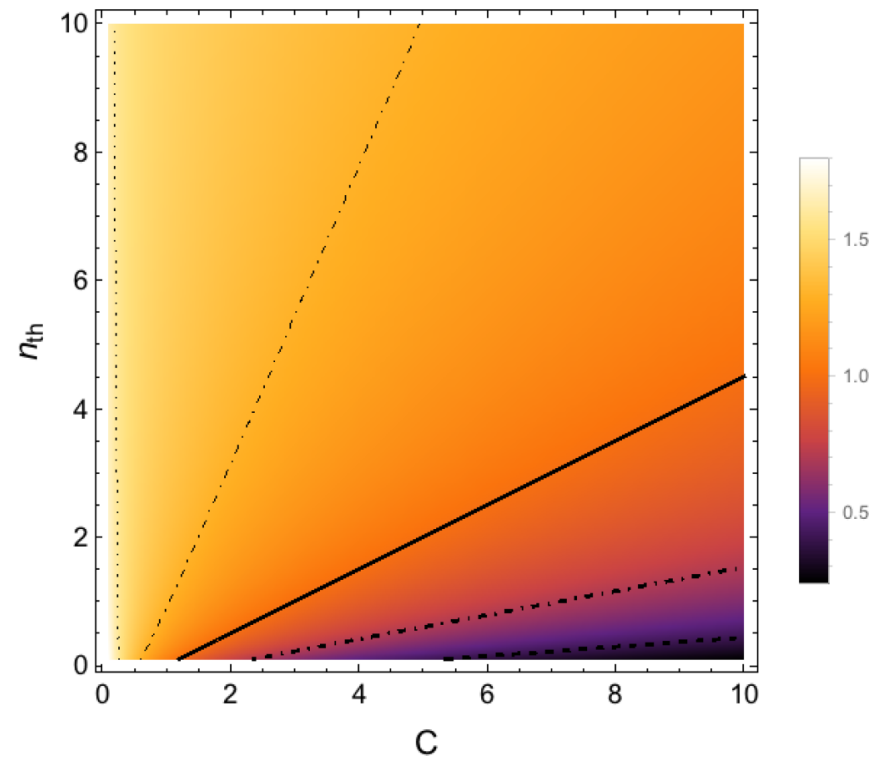

FIG. 5. Steady-state second-order correlation function $g^{(2)}(0)$ of the mechanical oscillator as a function of both the multiphoton cooperativity and the thermal occupation number. The various lines show contours of constant $g^{(2)}(0): g^{(2)}(0)=1.6($ dotted line $), g^{(2)}(0)=1.3$ (dot-dashed line), $g^{(2)}(0)=1.0$ (thick solid line), $g^{(2)}(0)=0.7$ (thick dot-dashed line), and $g^{(2)}(0)=0.4$ (thick dashed line).

phonon number

$$
n_{\mathrm{ss}}=\frac{\bar{n}_{\mathrm{th}}}{2 \bar{n}_{\mathrm{th}}+1},
$$

and the second-correlation function becomes unity. This situation is indicated by the thick solid line in Fig. 5: Regions of parameter space above this line yield steady-state bunching, whereas below this line antibunching is realized. Figure 6 shows three representative plots of the phonon number distributions of the mechanics, indicating that only the ground and first excited states are significantly populated if $C \gg \bar{n}_{\text {th }}+1$ (blue rhombi), the distribution being Poissonian

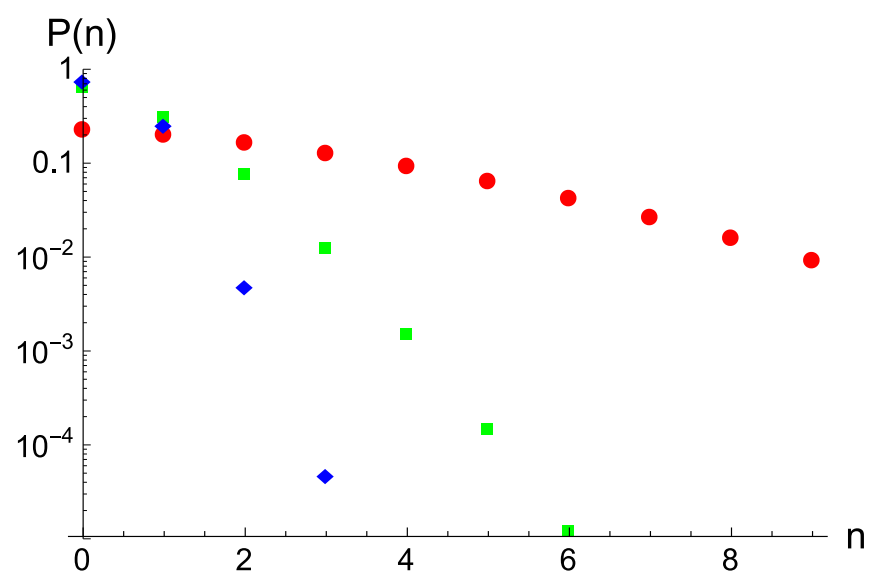

FIG. 6. Steady-state phonon distributions $P(n)$ of the mechanical oscillator with different multiphoton cooperativities at the same temperature, $\bar{n}_{\mathrm{th}}=20$ : $C=1$ (red circles), $C=41$ (green squares), and $C=1000$ (blue rhombi). if $C=2 \bar{n}_{\text {th }}+1$ (green circles) and the distribution becoming nearly exponential if $C \ll 2 \bar{n}_{\text {th }}+1$ (red circles).

We finish by noting that in the regime where the mechanical heat bath is at zero temperature thermal effects are completely negligible compared to quantum fluctuations, $\bar{n}_{\text {th }}=0$, and the diffusion matrix $D(\chi)$ reads

$$
D(\chi)=\left(\begin{array}{cc}
-C \mu^{2} & 0 \\
0 & -C v^{2}
\end{array}\right) .
$$

This situation was previously studied extensively in the context of quantum optics [45] and the steady-state complex $P$ distribution is given by

$$
P_{s}(\mu, \nu)=\frac{1-\frac{1}{C}}{2 \pi^{2}} e^{2 \mu \nu} \sum_{r=0}^{\infty} \frac{(-2 \mu \nu)^{r-1}}{\left(r+1-\frac{1}{C}\right) r !} .
$$

In this case the mechanical oscillator is coupled to an optical reservoir at zero temperature by the nonlinear optomechanical coupling and is also coupled to the mechanical heat bath at zero temperature by the intrinsic linear interaction. Then the steady state of the mechanical oscillator is the motional ground state, as expected, and thus the mean phonon number $n_{\mathrm{ss}}=0$ and the second-order correlation function $g^{(2)}(0)=0$ [45].

\section{EXPERIMENTAL FEASIBILITY}

There are of course barriers to realizing antibunching of a phonon field, but recent developments make this more feasible. Consider, for concreteness, the photonic crystal optomechanical cavity described in Ref. [47], in which a mechanical mode with effective mass $m=3.7 \mathrm{pg}$ and natural frequency $\omega_{m}=2 \pi \times 8.7 \mathrm{MHz}$ is coupled to an optical field via the quadratic optomechanical interaction with single-photon quadratic coupling coefficient $g_{0}=2 \pi \times 245 \mathrm{~Hz}$. This optomechanical system operating with intracavity photon numbers $n_{c}=10^{3}$, cavity decay rate $\kappa=2 \pi \times 5 \mathrm{GHz}$, and mechanical quality factor $Q_{m}=10^{7}$ and at temperature $T=11 \mathrm{mK}$ gives the cavity-enhanced optomechanical coupling strength $g=2 \pi \times 245 \mathrm{kHz}$, multiphoton cooperativity $C=110$, and thermal occupation number $\bar{n}_{\text {th }}=22$. This example comes close to realizing the conditions for observing antibunching but falls short in that it does not operate in the resolvedsideband regime since $\kappa \gg \omega_{m}$. On the other hand, operation of a photonic crystal optomechanical cavity in the resolvedsideband regime has previously been reported in a different setup $[6,48]$. Thus, even though no single experimental setup currently satisfies all the requirements needed for realizing an antibunched phonon field, this may be remedied in the near-future due to recent advances in microfabrication and nanotechnology, which have led to an optical resonator with a quality factor of order $10^{7}$ [49] and a mechanical oscillator with $Q_{m}=10^{8}$ [50]. In addition, the demonstration of a HanburyBrown-Twiss-type experiment [51] for a phonon field in a nanomechanical resonator paves the way to measuring the second-order correlation.

\section{SUMMARY AND CONCLUSIONS}

We have analytically investigated the steady state of a mechanical oscillator coupled to a single-mode optical field 
via a quadratic optomechanical interaction and in the weakcoupling limit. The mechanics was shown to experience an effective cubic nonlinearity in the limit that the cavity dissipation rate is much larger than both the optomechanical coupling and the mechanical damping rates, allowing for adiabatic elimination of the cavity field. Our key result is that the steady-state phonon field is chaotic if the multiphoton cooperativity obeys $C<2 \bar{n}_{\text {th }}+1$, whereas it is antibunched if $C>2 \bar{n}_{\text {th }}+1$. Thus our calculation opens the door to control of the second-order correlation of the mechanical oscillator in the weak-coupling regime and the observation of phonon antibunching.

\section{ACKNOWLEDGMENT}

This work was supported by National Research Foundation of Korea (NRF) Grant No. 2015R1C1A1A01052349 funded by the Korea government (MSIP).

\section{APPENDIX: STEADY-STATE COMPLEX $P$ DISTRIBUTION IN THE LOW-TEMPERATURE REGIME}

In order to find the steady-state complex $P$ distribution of the mechanics, we follow the procedures outlined in Ref. [45]. Equation (24) can be written as

$$
\begin{aligned}
\frac{d P}{d \tau}= & \frac{\partial}{\partial \mu}\left[\frac{\mu}{2}+C \mu^{2} v-\frac{C}{2} \frac{\partial}{\partial \mu} \mu^{2}+\frac{\bar{n}_{\mathrm{th}}}{2} \frac{\partial}{\partial v}\right] P \\
& +\frac{\partial}{\partial v}\left[\frac{v}{2}+C v^{2} \mu-\frac{C}{2} \frac{\partial}{\partial v} v^{2}+\frac{\bar{n}_{\mathrm{th}}}{2} \frac{\partial}{\partial \mu}\right] P .
\end{aligned}
$$

The steady-state complex $P$ distribution can in general be obtained from

$$
\begin{aligned}
& {\left[\frac{\mu}{2}+C \mu^{2} v-\frac{C}{2} \frac{\partial}{\partial \mu} \mu^{2}+\frac{\bar{n}_{\mathrm{th}}}{2} \frac{\partial}{\partial v}\right] P_{s}=f(v),} \\
& {\left[\frac{v}{2}+C v^{2} \mu-\frac{C}{2} \frac{\partial}{\partial v} v^{2}+\frac{\bar{n}_{\mathrm{th}}}{2} \frac{\partial}{\partial \mu}\right] P_{s}=g(\mu),}
\end{aligned}
$$

where $f(v)$ and $g(\mu)$ must satisfy generalized potential conditions [45]. To find the form of these functions we write $P_{s}(\mu, v)$ as

$$
P_{s}(\mu, v)=\frac{Q(\mu, v)}{\left(C \mu \nu-\bar{n}_{\mathrm{th}}\right)^{2}} ;
$$

then Eqs. (A2) and (A3) can be written as

$$
\begin{aligned}
& \frac{\partial R(\mu, v)}{\partial \mu}=e^{-2 \mu \nu}\left(C \mu \nu-\bar{n}_{\mathrm{th}}\right)^{1-\frac{1+2 \bar{n}_{\mathrm{th}}}{C}} F(\mu, \nu), \\
& \frac{\partial R(\mu, \nu)}{\partial v}=e^{-2 \mu v}\left(C \mu \nu-\bar{n}_{\mathrm{th}}\right)^{1-\frac{1+2 \bar{n}_{\mathrm{th}}}{C}} G(\mu, \nu),
\end{aligned}
$$

where we define, for typographical simplicity,

$$
\begin{gathered}
R(\mu, v)=e^{-2 \mu \nu}\left(C \mu \nu-\bar{n}_{\mathrm{th}}\right)^{-\frac{1+2 \bar{n}_{\mathrm{th}}}{C}} Q(\mu, \nu), \\
F(\mu, v)=-2 \frac{C v^{2} f(v)+\bar{n}_{\mathrm{th}} g(\mu)}{C \mu \nu+\bar{n}_{\mathrm{th}}}, \\
G(\mu, v)=-2 \frac{C \mu^{2} g(\mu)+\bar{n}_{\mathrm{th}} f(\nu)}{C \mu \nu+\bar{n}_{\mathrm{th}}} .
\end{gathered}
$$

The generalized potential condition

$$
\frac{\partial^{2} R(\mu, \nu)}{\partial \nu \partial \mu}=\frac{\partial^{2} R(\mu, \nu)}{\partial \mu \partial \nu}
$$

can be written as

$$
\begin{aligned}
& \frac{\partial}{\partial v}\left[e^{-2 \mu \nu}\left(C \mu \nu-\bar{n}_{\mathrm{th}}\right)^{1-\frac{1+2 \bar{n}_{\mathrm{th}}}{C}} F(\mu, v)\right] \\
& \quad=\frac{\partial}{\partial \mu}\left[e^{-2 \mu \nu}\left(C \mu \nu-\bar{n}_{\mathrm{th}}\right)^{1-\frac{1+2 \bar{n}_{\mathrm{th}}}{C}} G(\mu, v)\right] .
\end{aligned}
$$

Equation (A11) is satisfied for

$$
\begin{aligned}
& f(v)=\frac{A}{v}, \\
& g(\mu)=\frac{A}{\mu},
\end{aligned}
$$

where $A$ is a constant. Thus, the steady-state complex $P$ distribution, after some algebra, is given by

$$
P_{s}(\mu, \nu)=e^{2 \mu \nu}\left(C \mu \nu-\bar{n}_{\mathrm{th}}\right)^{\frac{1+2 \bar{n}_{\mathrm{th}}}{C}-2}[B+A I(\mu, \nu)],
$$

where $B$ is a constant of integration and $I(\mu, v)$ is the indefinite integral

$$
I(\mu, \nu)=-2 \int d \mu \frac{e^{-2 \mu \nu}}{\mu}\left(C \mu \nu-\bar{n}_{\mathrm{th}}\right)^{1-\frac{1+2 \bar{n}_{\mathrm{th}}}{C}} .
$$

This integral may be calculated using a power-series expansion of the exponential function and the resulting steady-state complex $P$ distribution reads

$$
\begin{aligned}
P_{s}(\mu, \nu)= & B e^{2 \mu \nu}\left(C \mu \nu-\bar{n}_{\mathrm{th}}\right)^{\frac{1+2 \bar{n}_{\mathrm{th}}}{C}}-2 \\
& +\frac{2 A e^{2 \mu \nu}}{\left(1+2 \bar{n}_{\mathrm{th}}-C\right) \mu \nu}{ }_{2} F_{1}\left(1,1 ; \frac{1+2 \bar{n}_{\mathrm{th}}}{C} ; \frac{\bar{n}_{\mathrm{th}}}{C \mu \nu}\right) \\
& +\frac{2 A e^{2 \mu \nu}}{\bar{n}_{\mathrm{th}}} \sum_{r=1}^{\infty} \frac{(-2 \mu \nu)^{r}}{r r !} \\
& \times{ }_{2} F_{1}\left(1,2+r-\frac{1+2 \bar{n}_{\mathrm{th}}}{C} ; 1+r ; \frac{C \mu \nu}{\bar{n}_{\mathrm{th}}}\right) .
\end{aligned}
$$

It should be noted that the two constants $A$ and $B$ are chosen from the normalization condition and the requirement that the phonon number distribution be nonnegative. Using the complex $P$ distribution function, all normal-ordered moments at steady state can be obtained from

$$
\left\langle\left(\hat{b}^{\dagger}\right)^{n}(\hat{b})^{n^{\prime}}\right\rangle_{\mathrm{ss}}=\int d \mu d \nu(\nu)^{n}(\mu)^{n^{\prime}} P_{s}(\mu, \nu) .
$$

Making the change of variables

$$
\begin{gathered}
N=\mu \nu, \\
z=\mu
\end{gathered}
$$

and choosing a circular contour around the origin for the $z$-line integral and a Hankel contour for the $N$-line integral [52], one can find the normalization condition, the mean phonon number, the second-order correlation, and so on, from Eq. (A17). 
The normalization condition reads

$$
\begin{aligned}
\frac{1}{4 \pi^{2}}= & -B \frac{e^{\frac{2 \bar{n}_{\mathrm{th}}}{C}}}{2 \Gamma\left(2-\frac{1+2 \bar{n}_{\mathrm{th}}}{C}\right)}\left(\frac{C}{2}\right)^{\frac{1+2 \bar{n}_{\mathrm{th}}}{C}-2} \\
& -A \frac{2 \Gamma\left(\frac{1+2 \bar{n}_{\mathrm{th}}}{C}\right)}{1+2 \bar{n}_{\mathrm{th}}-C} \sum_{k=0}^{\infty} \frac{\left(2 \bar{n}_{\mathrm{th}} / C\right)^{k}}{\Gamma\left(\frac{1+2 \bar{n}_{\mathrm{th}}}{C}+k\right)} .
\end{aligned}
$$

The populations of the $m$ th number state are given by

$$
\begin{aligned}
P_{m}= & -B \sum_{k=0}^{m} \frac{4 \pi^{2} e^{\frac{\bar{n}_{\mathrm{th}}}{C}} C^{\frac{1+2 \bar{n}_{\mathrm{th}}}{C}}-2\left(\frac{\bar{n}_{\mathrm{th}}}{C}\right)^{m-k}}{\Gamma(m-k+1) \Gamma(k+1) \Gamma\left(2-k-\frac{1+2 \bar{n}_{\mathrm{th}}}{C}\right)} \\
& -A \frac{8 \pi^{2} \Gamma\left(\frac{1+2 \bar{n}_{\mathrm{th}}}{C}\right)}{\left(1+2 \bar{n}_{\mathrm{th}}-C\right) m !} \\
& \times \sum_{k=m}^{\infty} \frac{\Gamma(k+1)\left(\bar{n}_{\mathrm{th}} / C\right)^{k}}{\Gamma\left(\frac{1+2 \bar{n}_{\mathrm{th}}}{C}+k\right) \Gamma(k+1-m)} .
\end{aligned}
$$

In order for the phonon number distribution to be nonnegative, $B=0$ for $C \neq 1+2 \bar{n}_{\text {th }}$ and $A=0$ for $C=1+2 \bar{n}_{\text {th }}$ due to the oscillatory behavior of the $\Gamma$ function.
If $C \neq 1+2 \bar{n}_{\text {th }}$, the normalization constant $A$ is given by

$$
A=-\frac{1+2 \bar{n}_{\mathrm{th}}-C}{8 \pi^{2} \Gamma\left(\frac{1+2 \bar{n}_{\mathrm{th}}}{C}\right) \sum_{k=0}^{\infty} \frac{\left(2 \bar{n}_{\mathrm{th}} / C\right)^{k}}{\Gamma\left(\frac{1+2 \bar{n}_{\mathrm{th}}}{C}+k\right)}} .
$$

The mean phonon number is given by

$$
n_{\mathrm{ss}}=\frac{1}{2} \frac{\sum_{k=0}^{\infty} \frac{k}{\Gamma\left(\frac{1+2 \bar{n}_{\text {th }}}{C}+k\right)}\left(\frac{2 \bar{n}_{\text {th }}}{C}\right)^{k}}{\sum_{k=0}^{\infty} \frac{1}{\Gamma\left(\frac{1+2 \bar{n}_{\text {th }}}{C}+k\right)}\left(\frac{2 \bar{n}_{\text {th }}}{C}\right)^{k}},
$$

and the second-order correlation function $g^{(2)}(0)$ is

$$
g^{(2)}(0)=\frac{\sum_{k, k \prime}^{\infty} \frac{k(k-1)}{\Gamma\left(\frac{1+2 \bar{n}_{\mathrm{th}}}{C}+k\right) \Gamma\left(\frac{1+2 \bar{n}_{\mathrm{th}}}{C}+k^{\prime}\right)}\left(\frac{2 \bar{n}_{\mathrm{th}}}{C}\right)^{k+k^{\prime}}}{\sum_{k, k^{\prime}}^{\infty} \frac{k k^{\prime}}{\Gamma\left(\frac{1+2 \bar{n}_{\mathrm{th}}}{C}+k\right) \Gamma\left(\frac{1+2 \bar{n}_{\mathrm{th}}}{C}+k^{\prime}\right)}\left(\frac{2 \bar{n}_{\mathrm{th}}}{C}\right)^{k+k^{\prime}}} .
$$

[1] M. Aspelmeyer, T. J. Kippenberg, and F. Marquardt, Rev. Mod. Phys. 86, 1391 (2014).

[2] P. Meystre, Ann. Phys. 525, 215 (2013).

[3] G. J. Milburn and M. J. Woolley, Acta Phys. Slov. 61, 483 (2011).

[4] W. P. Bowen and G. J. Milburn, Quantum Optomechanics (CRC Press, Boca Raton, FL, 2016).

[5] J. D. Teufel et al., Nature 475, 359 (2011).

[6] J. Chan et al., Nature 478, 89 (2011).

[7] S. Weis et al., Science 330, 1520 (2010).

[8] E. Verhagen, S. Deléglise, S. Weis, A. Schliesser, and T. J. Kippenberg, Nature 482, 63 (2012).

[9] T. A. Palomaki, J. W. Harlow, J. D. Teufel, R. W. Simmonds, and K. W. Lehnert, Nature 495, 210 (2013).

[10] T. A. Palomaki, J. D. Teufel, R. W. Simmonds, and K. W. Lehnert, Science 342, 710 (2013).

[11] A. B. Shkarin, N. E. Flowers-Jacobs, S. W. Hoch, A. D. Kashkanova, C. Deutsch, J. Reichel, and J. G. E. Harris, Phys. Rev. Lett. 112, 013602 (2014).

[12] P. Verlot, A. Tavernarakis, T. Briant, P.-F. Cohadon, and A. Heidmann, Phys. Rev. Lett. 102, 103601 (2009).

[13] B. P. Abbott et al., Phys. Rev. Lett. 116, 061102 (2016).

[14] I. Pikovski, M. R. Vanner, M. Aspelmeyer, M. S. Kim, and Č. Brukner, Nat. Phys. 8, 393 (2012).

[15] B. Vogell, K. Stannigel, P. Zoller, K. Hammerer, M. T. Rakher, M. Korppi, A. Jöckel, and P. Treutlein, Phys. Rev. A 87, 023816 (2013).

[16] B. Vogell et al., New J. Phys. 17, 043044 (2015).

[17] D. A. Golter, T. Oo, M. Amezcua, K. A. Stewart, and H. Wang, Phys. Rev. Lett. 116, 143602 (2016).

[18] R. W. Boyd, Nonlinear Optics, 3rd ed. (Elsevier, Burlington, MA, 2008).

[19] P. Rabl, Phys. Rev. Lett. 107, 063601 (2011).
[20] A. Nunnenkamp, K. Borkje, and S. M. Girvin, Phys. Rev. Lett. 107, 063602 (2011).

[21] D. A. Rodrigues and A. D. Armour, Phys. Rev. Lett. 104, 053601 (2010).

[22] J. Qian, A. A. Clerk, K. Hammerer, and F. Marquardt, Phys. Rev. Lett. 109, 253601 (2012).

[23] P. D. Nation, Phys. Rev. A 88, 053828 (2013).

[24] N. Lörch, J. Qian, A. A. Clerk, F. Marquardt, and K. Hammerer, Phys. Rev. X 4, 011015 (2014).

[25] M. Ludwig, A. H. Safavi-Naeini, O. Painter, and F. Marquardt, Phys. Rev. Lett. 109, 063601 (2012).

[26] X. Xu, M. Gullans, and J. M. Taylor, Phys. Rev. A 91, 013818 (2015).

[27] J. Li, A. Xuereb, N. Malossi, and D. Vitali, J. Opt. 18, 084001 (2016)

[28] N. Lörch and K. Hammerer, Phys. Rev. A 91, 061803(R) (2015).

[29] S. Rips, M. Kiffner, I. Wilson-Rae, and M. J. Hartmann, New J. Phys. 14, 023042 (2012).

[30] M. Grimm, C. Bruder, and N. Lörch, J. Opt. 18, 094004 (2016).

[31] X. Wang, A. Miranowicz, H. R. Li, and F. Nori, Phys. Rev. A 93, 063861 (2016).

[32] X. Wang, A. Miranowicz, H. R. Li, and F. Nori, Phys. Rev. A 94, 053858 (2016).

[33] J. D. Thompson et al., Nature 452, 72 (2008).

[34] J. C. Sankey et al., Nat. Phys. 6, 707 (2010).

[35] H. Seok, L. F. Buchmann, S. Singh, and P. Meystre, Phys. Rev. A 86, 063829 (2012).

[36] P. Meystre and M. Sargent III, Elements of Quantum Optics, 4th ed. (Springer, Berlin, 2007).

[37] D. F. Walls and G. J. Milburn, Quantum Optics, 2nd ed. (Springer, Berlin, 2008).

[38] H. J. Carmichael, Statistical Methods in Quantum Optics 2 (Springer, Berlin, 2008).

[39] G. P. Hildred, Opt. Acta 27, 1621 (1980). 
[40] V. V. Dodonov and S. S. Mizrahi, J. Phys. A 30, 5657 (1997).

[41] P. D. Drummond and C. W. Gardiner, J. Phys. A 13, 2353 (1980).

[42] P. D. Drummond and D. F. Walls, J. Phys. A 13, 725 (1980).

[43] P. D. Drummond, K. J. McNeil, and D. F. Walls, Opt. Acta 28, 211 (1981).

[44] C. W. Gardiner and P. Zoller, Quantum Noise, 3rd ed. (Springer, Berlin, 2010).

[45] A. M. Smith and C. W. Gardiner, Phys. Rev. A 39, 3511 (1989).

[46] A. Nunnenkamp, K. Børkje, J. G. E. Harris, and S. M. Girvin, Phys. Rev. A 82, 021806(R) (2010).
[47] T. K. Paraïso, M. Kalaee, L. Zang, H. Pfeifer, F. Marquardt, and O. Painter, Phys. Rev. X 5, 041024 (2015).

[48] S. M. Meenehan, J. D. Cohen, G. S. MacCabe, F. Marsili, M. D. Shaw, and O. Painter, Phys. Rev. X 5, 041002 (2015).

[49] H. Sekoguchi, Y. Takahashi, T. Asano, and S. Noda, Opt. Express 22, 916 (2014).

[50] C. Reinhardt, T. Müller, A. Bourassa, and J. C. Sankey, Phys. Rev. X 6, 021001 (2016).

[51] J. D. Cohen et al., Nature 520, 522 (2015).

[52] T. M. MacRobert, Functions of a Complex Variable (Leopold Classic Library, Kiev, Ukraine, 2016). 